\title{
Correlation analysis between four serum biomarkers of liver fibrosis and liver function in infants with cholestasis
}

\author{
NING TANG ${ }^{1}$, YAPING ZHANG ${ }^{1}$, ZEYU LIU $^{1}$, TAO FU $^{2}$, QINGHONG LIANG $^{1}$ and XUEMEI AI ${ }^{1}$ \\ ${ }^{1}$ Department of Pediatrics, The Third Hospital of Hebei Medical University, Shijiazhuang, Hebei 050051; \\ ${ }^{2}$ Worker's Hospital of China Electronics Technology Group Corporation 54, Shijiazhuang, Hebei 050081, P.R. China
}

Received February 25, 2016; Accepted May 12, 2016

DOI: $10.3892 /$ br.2016.681

\begin{abstract}
The aim of the present study was to investigate the correlation between four serum biomarkers of liver fibrosis and liver function in infants with cholestasis. A total of 30 infants with cholestasis and 20 healthy infants were included in the study. Biochemical assays based on the initial rate method and colorimetric assays were conducted to determine the levels of liver function markers in the serum [such as alanine aminotransferase (ALT), aspartate aminotransferase (AST), total bilirubin (TBIL), direct bilirubin (DBIL), indirect bilirubin (IBIL), $\gamma$-glutamyl transferase $(\gamma-\mathrm{GT})$, cholinesterase (CHE) and total bile acids (TBA)] and four serum biomarkers of liver fibrosis were measured using radioimmunoassays [hyaluronic acid (HA), procollagen type III (PCIII), laminin (LN) and collagen type IV (cIV)]. The serum levels of ALT, AST, TBIL, DBIL, IBIL, $\gamma$-GT and TBA in the infants with cholestasis were significantly higher compared to the healthy infants $(\mathrm{P}<0.01)$; the serum levels of CHE in the infants with cholestasis were significantly lower compared to the healthy infants $(\mathrm{P}<0.01)$. The serum levels of HA, PCIII, and cIV in the infants with cholestasis were significantly higher compared to the healthy infants $(\mathrm{P}<0.01)$. Correlation analyses between liver function and the four biomarkers of liver fibrosis showed that HA was positively correlated with AST and $\gamma$-GT $(\mathrm{P}<0.05)$ and negatively correlated with ALT, CHE and TBA $(\mathrm{P}<0.05)$. cIV was positively correlated with $\gamma$-GT $(\mathrm{P}<0.05)$ and negatively correlated with CHE $(\mathrm{P}<0.05)$. In conclusion, statistically significant differences were identified for the liver function markers (ALT, AST, TBIL, DBIL, IBIL, $\gamma$-GT and TBA) and the biomarkers HA, PCIII and cIV of liver fibrosis between infants with cholestasis and healthy infants. Thus, the serum
\end{abstract}

Correspondence to: Dr Yaping Zhang, Department of Pediatrics, The Third Hospital of Hebei Medical University, 139 Ziqiang Road, Shijiazhuang, Hebei 050051, P.R. China

E-mail: yapingzhang@aliyun.com

Key words: infant, cholestasis, liver function markers, biomarkers, liver fibrosis, correlation analysis levels of HA, cIV, $\gamma$-GT and CHE are sensitive markers for cholestatic liver fibrosis in infants.

\section{Introduction}

The incidence of infants with cholestasis is between 1 in 2,500 and 1 in 5,000 newborns worldwide and has become the leading cause of hospitalization of children with liver diseases. A variety of factors, such as infection, poisoning, autoimmune issues, genetic defects, metabolic abnormalities and other uncertainties, can trigger dysfunction of hepatocytes, reduction of bile flow, or bile duct obstruction, which may result in cholestasis (1-3). Delayed treatment may affect the development of children and result in malnutrition. Part of the liver may turn fibrotic, resulting in cholestatic cirrhosis (4). For a long time, the degree of liver fibrosis could only be determined through liver biopsy. Non-invasive examination and assessment of liver fibrosis have been a focus of research in recent years. To date, non-invasive assessments of cholestasis in infants mainly involve four marker liver fibrosis assays and liver function assays. Correlation analyses between the liver fibrosis assays and the liver function assays have been rarely reported. The present study aimed to provide a simple, safe and economical method for assessing liver fibrosis in infants and promote an effective method for early diagnosis and clinical monitoring of liver fibrosis in infants.

\section{Subjects and methods}

Research subjects. Thirty infants (experimental group) with cholestasis were recruited from the outpatient clinic and admitted to the Department of Pediatrics at The Third Hospital of Hebei Medical University (Hebei, China) between May 2012 and December 2013. Twenty healthy infants who underwent a routine examination at The Third Hospital of Hebei Medical University were included in the control group of this study. The grouping was not adjusted for gender. All infants were $\leq 18$ months of age. Inclusion criteria of infants with cholestasis were: i) $\leq 18$ months of age, with morbidity within 6 months after birth; ii) diagnosis of infant cholestasis based on the recommendation of the American Academy of Pediatrics; i.e., jaundice in full-term infants with total bilirubin (TBIL) $<85 \mu \mathrm{mol} / 1$ and direct bilirubin (DBIL) $>17 \mu \mathrm{mol} / \mathrm{l}$; 
or TBIL $>85 \mu \mathrm{mol} / 1$ and DBIL levels $>20 \%$ of TBIL (5). The study was approved by the medical ethics committee of The Third Hospital of Hebei Medical University. Parents of all the infants signed the written informed consent prior to entering the study.

\section{Methods}

Four serum biomarkers for the determination of liver fibrosis. Radioimmunoassays were used to detect four serum biomarkers [hyaluronic acid (HA), procollagen type III (PCIII), laminin (LN), and collagen type IV (cIV)] of liver fibrosis. Infants were required to fast overnight and 2-3 ml of venous blood was collected in the early morning. All the blood samples were placed in pro-coagulant tubes and centrifuged at $1,006 \mathrm{x}$ g for $3 \mathrm{~min}$ to isolate the serum for later assays. For blood samples that could not be measured on the day of collection, serum was isolated and stored at $-20^{\circ} \mathrm{C}$, and measurements were completed within a week. The four biomarkers of liver fibrosis were tested using radioimmunoassays from Beijing North Institute Biotechnology (Beijing, China) and measured with a $\gamma$-radioimmunoassay counter (XH-6020; Xi'an Nuclear Instrument Factory, Shaanxi, China). Experimental procedures strictly followed the manufacturer's protocols.

Measurement of liver function in the serum. Detection kits for liver function biomarkers [alanine aminotransferase (ALT), aspartate aminotransferase (AST), TBIL, DBIL, indirect bilirubin (IBIL), $\gamma$-glutamyl transferase $(\gamma$-GT), cholinesterase (CHE) and total bile acids (TBA)] were purchased from Johnson \& Johnson Medical (Shanghai), Ltd. (Shanghai, China). An automatic biochemical analyzer (AU5400TM; Olympus Optical Corp., Ltd., Japan) was used for measurements. Approximately 2-3 ml of venous blood from each patient were collected in the early morning after fasting overnight and placed in a pro-coagulant tube, followed by centrifuging at $1,006 \mathrm{x}$ g for $3 \mathrm{~min}$ to isolate serum for further assays. Measurements of ALT, AST, $\gamma$-GT, CHE and TBA were based on assays using the initial rate method and measurements of TBIL, DBIL and IBIL were based on colorimetric assays.

Statistical analysis. SPSS 19.0 software (SPSS, Inc., Armonk, NY, USA) was used for data processing and statistical analysis. Statistical results are presented as mean \pm standard deviation. Normally distributed numerical data were compared using an independent sample t-test. Comparisons between non-normally distributed numerical data were performed with a rank sum test. Differences in each liver function assay and each of the four biomarkers for liver fibrosis were measured by partial correlation analysis. $\mathrm{P}<0.05$ was considered to indicate a statistically significant difference.

\section{Results}

Results of liver function tests in infants with cholestasis. Serum levels of ALT, AST, TBIL, DBIL, IBIL, $\gamma$-GT and TBA in the infants with cholestasis were significantly higher compared to the healthy infants in the control group $(\mathrm{P}<0.01$
Table I. Changes of liver function indexes in infants with cholestasis.

\begin{tabular}{lccc}
\hline Variables & $\begin{array}{c}\text { Control } \\
\text { group }\end{array}$ & $\begin{array}{c}\text { Infants with } \\
\text { cholestasis }\end{array}$ & P-value \\
\hline Total, $\mathrm{n}$ & 20 & 30 & \\
ALT, U/l & $11.900 \pm 1.917$ & $117.900 \pm 261.894$ & $<0.01$ \\
AST, U/1 & $29.450 \pm 3.602$ & $115.567 \pm 208.786$ & $<0.01$ \\
TBIL, $\mu \mathrm{mol} / 1$ & $7.140 \pm 1.632$ & $103.970 \pm 74.226$ & $<0.01$ \\
DBIL, $\mu \mathrm{mol} / 1$ & $2.165 \pm 0.555$ & $55.057 \pm 41.516$ & $<0.01$ \\
IBIL, $\mu \mathrm{mol} / 1$ & $4.975 \pm 1.212$ & $48.247 \pm 47.440$ & $<0.01$ \\
$\gamma$-GT, U/1 & $11.000 \pm 1.487$ & $169.300 \pm 184.600$ & $<0.01$ \\
CHE, KU/1 & $9.601 \pm 1.171$ & $6.639 \pm 1.936$ & $<0.01$ \\
TBA, $\mu \mathrm{mol} / 1$ & $3.195 \pm 2.587$ & $49.447 \pm 58.214$ & $<0.01$ \\
\hline
\end{tabular}

ALT, alanine aminotransferase; AST, aspartate aminotransferase; TBIL, total bilirubin; DBIL, direct bilirubin; IBIL, indirect bilirubin; $\gamma$-GT, $\gamma$-glutamyl transferase; CHE, cholinesterase; TBA, total bile acids.

Table II. Variation of the four indicators of liver fibrosis in infants with cholestasis.

\begin{tabular}{lccc}
\hline Variables & $\begin{array}{c}\text { Control } \\
\text { group }\end{array}$ & $\begin{array}{c}\text { Infants with } \\
\text { cholestasis }\end{array}$ & P-value \\
\hline Total, n & 20 & 30 & \\
HA, ng/ml & $81.165 \pm 7.319$ & $201.273 \pm 177.030$ & $<0.01$ \\
PCIII, ng/ml & $141.314 \pm 26.609$ & $541.877 \pm 170.920$ & $<0.01$ \\
LN, ng/ml & $116.209 \pm 8.374$ & $123.637 \pm 19.322$ & $>0.05$ \\
cIV, ng/ml & $78.430 \pm 10.205$ & $134.371 \pm 44.984$ & $<0.01$ \\
\hline
\end{tabular}

HA, hyaluronic acid; PCIII, procollagen type III; LN, laminin; cIV, collagen type IV.

for each test); while serum levels of CHE in the infants with cholestasis were significantly lower compared to the healthy infants $(\mathrm{P}<0.01$; Table I).

Observation outcomes for the four biomarkers of liver fibrosis in infants with cholestasis. Among the four biomarkers of liver fibrosis, serum levels of HA, PCIII and cIV in the infants with cholestasis were significantly higher compared to the healthy infants $(\mathrm{P}<0.01$ for each test; Table II).

Correlation analyses between liver function tests and the four biomarkers of liver fibrosis in infants with cholestasis. HA was positively correlated with AST and $\gamma$-GT $(r=0.736$, $\mathrm{P}<0.01 ; \mathrm{r}=0.599, \mathrm{P}<0.01$, respectively; Figs. 1 and 2$)$ and negatively correlated with ALT, CHE and TBA $(r=-0.627$, $\mathrm{P}<0.01 ; \mathrm{r}=-0.427, \mathrm{P}<0.05 ; \mathrm{r}=-0.463, \mathrm{P}<0.05$, respectively; Figs. 3-5). cIV was positively correlated with $\gamma$-GT $(r=0.466$, $\mathrm{P}<0.05$; Fig. 6) and negatively correlated with $\mathrm{CHE}(\mathrm{r}=-0.520$, $\mathrm{P}<0.05$; Table III; Fig. 7). 


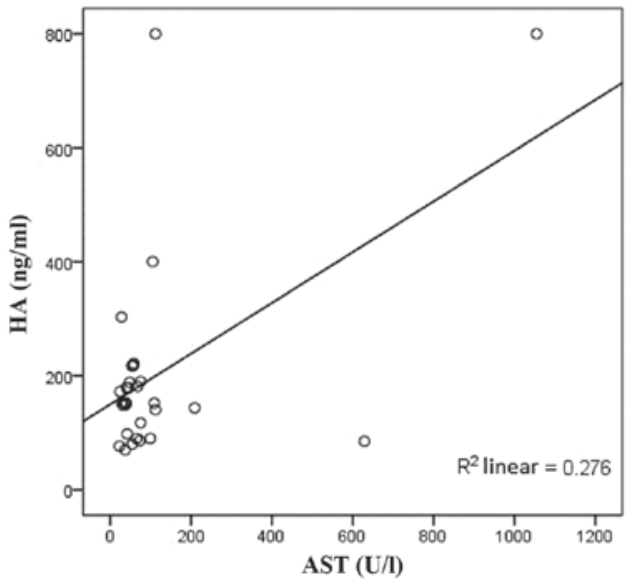

Figure 1. Hyaluronic acid (HA) and aspartate aminotransferase (AST) are positively associated in infantile cholestatic liver disease. $\mathrm{n}=30, \mathrm{r}=0.736, \mathrm{P}<0.01$.

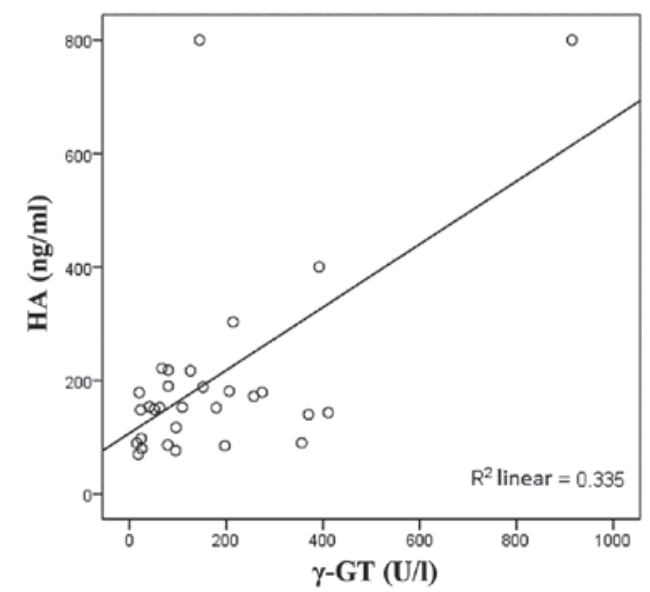

Figure 2. Hyaluronic acid (HA) and $\gamma$-glutamyl transferase $(\gamma$-GT) are positively related in infantile cholestatic liver disease. $\mathrm{n}=30, \mathrm{r}=0.599, \mathrm{P}<0.01$.

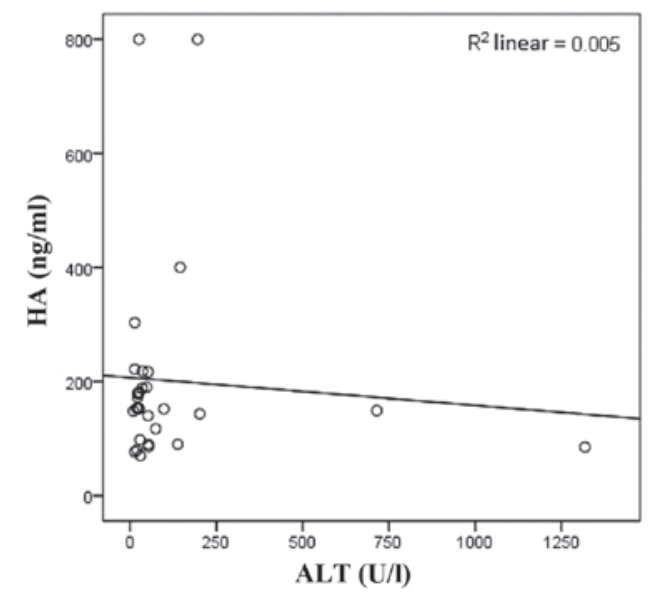

Figure 3. Hyaluronic acid (HA) and alanine aminotransferase (ALT) are negatively related in infantile cholestatic liver disease. $n=30, r=-0.627, \mathrm{P}<0.01$.

\section{Discussion}

Infants with cholestasis should be diagnosed early and receive effective treatment in a timely manner. Delayed treatment

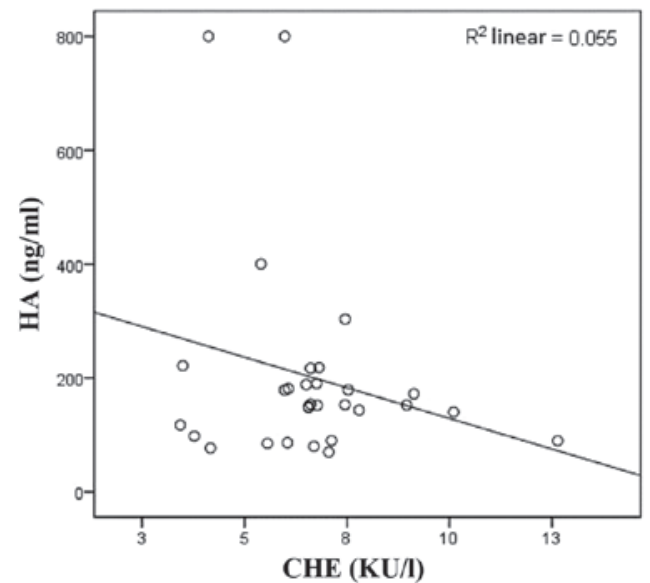

Figure 4. Hyaluronic acid (HA) and cholinesterase (CHE) are negatively related in infantile cholestatic liver disease. $\mathrm{n}=30, \mathrm{r}=-0.427, \mathrm{P}<0.05$.

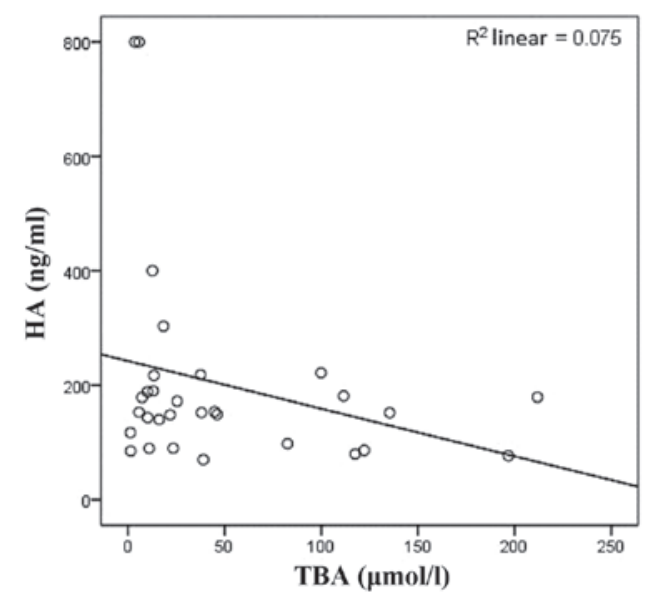

Figure 5. Hyaluronic acid (HA) and total bile acids (TBA) are negatively related in infantile cholestatic liver disease. $n=30, r=-0.463, P<0.05$.

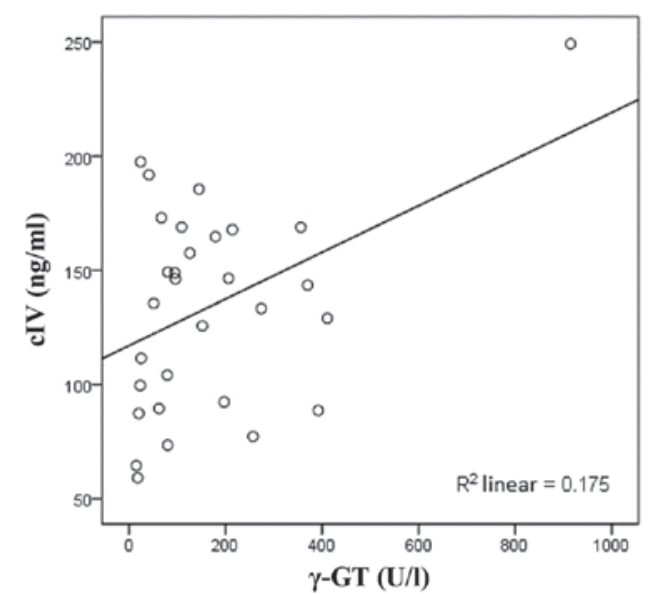

Figure 6. Collagen type IV (cIV) and $\gamma$-glutamyl transferase $(\gamma$-GT) are positively related in infantile cholestatic liver disease. $n=30, r=0.466, P<0.05$.

of cholestasis in infants seriously affects their development, resulting in liver fibrosis. Certain cases may further develop cholestatic cirrhosis. Therefore, early diagnosis of liver fibrosis in the infants with cholestasis becomes particularly important. 
Table III. Correlation analyses between liver function markers and the four biomarkers of liver fibrosis in infants with cholestasis.

\begin{tabular}{|c|c|c|c|c|c|c|c|c|}
\hline \multirow[b]{2}{*}{ Marker } & \multicolumn{2}{|c|}{$\mathrm{HA}, \mathrm{ng} / \mathrm{ml}$} & \multicolumn{2}{|c|}{ PCIII, ng/ml } & \multicolumn{2}{|c|}{$\mathrm{LN}, \mathrm{ng} / \mathrm{ml}$} & \multicolumn{2}{|c|}{$\mathrm{cIV}, \mathrm{ng} / \mathrm{ml}$} \\
\hline & $\mathrm{r}$ & P-value & $\mathrm{r}$ & P-value & $\mathrm{r}$ & P-value & $\mathrm{r}$ & P-value \\
\hline ALT, U/1 & -0.627 & $<0.01$ & 0.086 & N.S. & -0.350 & N.S. & -0.051 & N.S. \\
\hline AST, U/l & 0.736 & $<0.01$ & -0.220 & N.S. & 0.342 & N.S. & 0.020 & N.S. \\
\hline $\mathrm{TBIL}, \mu \mathrm{mol} / 1$ & 0.016 & N.S. & -0.019 & N.S. & -0.154 & N.S. & 0.374 & N.S. \\
\hline $\mathrm{DBIL}, \mu \mathrm{mol} / \mathrm{l}$ & -0.356 & N.S. & 0.031 & N.S. & 0.156 & N.S. & -0.356 & N.S. \\
\hline IBIL, $\mu \mathrm{mol} / 1$ & 0.028 & N.S. & 0.002 & N.S. & 0.168 & N.S. & -0.378 & N.S. \\
\hline$\gamma-\mathrm{GT}, \mathrm{U} / 1$ & 0.599 & $<0.01$ & -0.124 & N.S. & 0.237 & N.S. & 0.466 & $<0.05$ \\
\hline CHE, KU/l & -0.427 & $<0.05$ & 0.219 & N.S. & -0.189 & N.S. & -0.520 & $<0.05$ \\
\hline $\mathrm{TBA}, \mu \mathrm{mol} / 1$ & -0.463 & $<0.05$ & 0.236 & N.S. & -0.192 & N.S. & 0.077 & N.S. \\
\hline
\end{tabular}

ALT, alanine aminotransferase; AST, aspartate aminotransferase; TBIL, total bilirubin; DBIL, direct bilirubin; IBIL, indirect bilirubin; $\gamma$-GT, $\gamma$-glutamyl transferase; CHE, cholinesterase; TBA, total bile acids; HA, hyaluronic acid; PCIII, procollagen type III; LN, laminin; cIV, collagen type IV; N.S. not significant.

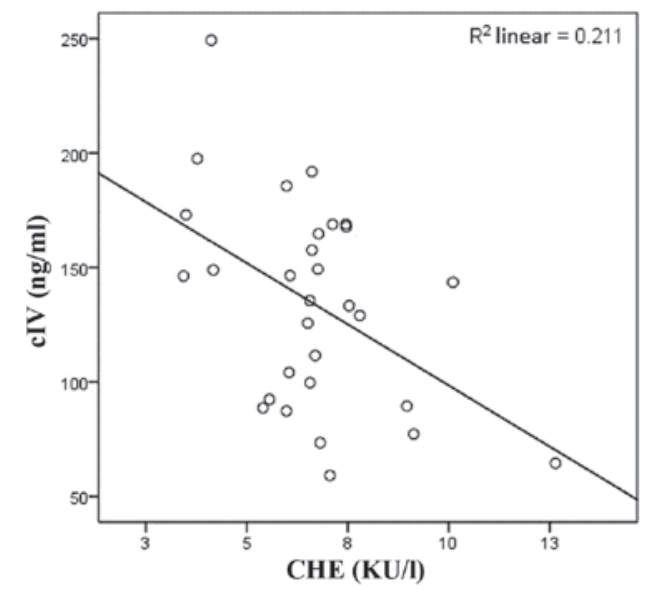

Figure 7. Collagen type IV (cIV) and cholinesterase (CHE) are negatively related in infantile cholestatic liver disease. $\mathrm{n}=30, \mathrm{r}=-0.520, \mathrm{P}<0.05$.

Serum HA, PCIII, cIV and LN are four common biomarkers of liver fibrosis. HA is one of the components of proteoglycan in the extracellular matrix (ECM) of hepatocytes. During liver tissue damage, the hepatic stellate cells (HSCs) synthesize HA and the level of HA increases. In addition, HA degradation in sinusoidal endothelial cells decreases, resulting in elevated levels of serum HA (6). The increased rate of serum HA in liver fibrosis was particularly associated with the severity of liver lesions. Serum HA can accurately and sensitively reflect the damages of hepatocytes and the amount of fibers in the liver (7-9). PCIII are type III collagen precursors and are significantly associated with the quantitation of type III procollagen peptide (PCIIIP). These indicators are valuable in the early diagnosis of liver fibrosis and significant in the prognosis of chronic liver disease. Serum levels of PCIII are consistent with the progression of liver fibrosis and activity. However, serum levels of PCIII have no specificity with liver fibrosis as they also increase in fibrosis of other organs. cIV is an important structural component of the reticular basement membrane. Following its synthesis in hepatocytes, cIV directly uses the procollagen form to participate in the composition of ECM. cIV content increases in the early stage of liver fibrosis, and its conversion rate accelerates during the progression of liver fibrosis. Eventually, cIV forms a complete basement membrane with the continuous deposition of LN. Clinical studies have shown that serum levels of cIV sensitively reflect damage in hepatocytes and the severity of liver fibrosis. Serum cIV is a biomarker for early liver fibrosis. LN is a structural glycoprotein and is mainly distributed in the transparent layer of the basement membrane (10). During liver cirrhosis, HSC synthesized LN levels are significantly increased, which is an important basis for the formation of portal hypertension. Clinical studies confirmed that serum levels of LN were positively correlated with the severity of liver cirrhosis. Detection of serum LN had an important diagnostic value for portal hypertension. In the present study, despite serum LN, serum levels of HA, PCIII and cIV in the infants with cholestasis were significantly higher compared to the healthy infants. However, the study was a clinical trial, which could not exclude differences in the course of disease, the living environment and individual infants, which may have affected the experimental results. These results demonstrated no significant differences in serum LN between the infants with cholestasis and the healthy infants. One possible explanation was that serum LN accumulation in an early stage of liver cirrhosis was not as significant as in later stages of liver cirrhosis. Thus, monitoring of serum LN at the early stage of liver cirrhosis had no significant clinical impact.

Liver biochemical tests are also known as liver function tests, which are important clinical tests to determine the presence of liver damage, to evaluate the severity of liver diseases, to track the progression of liver diseases, and to determine the treatment efficacies and prognoses of liver disease. Liver function tests in this study showed that levels of serum ALT, AST, TBIL, DBIL, IBIL, $\gamma$-GT, CHE and TBA in the infants with cholestasis were significantly higher compared to the healthy infants, which may be associated with different degrees of damage in the epithelial cells of bile ducts and hepatocytes 
following cholestasis, resulting in damage to normal physiological liver function and apoptosis.

In order to find simple assessments of liver fibrosis in infants and promote early diagnosis and clinical monitoring in infants with different degrees of liver fibrosis, the present study conducted correlation analyses between four serum biomarkers of liver fibrosis and liver function markers in infants with cholestasis. The results showed that serum HA was positively correlated with serum AST and negatively correlated with serum ALT. ALT is mainly present in the cytoplasm and is most abundantly located in liver cells. ALT is a sensitive marker for damage of hepatocytes. ASTs are mainly present in the cytoplasm and mitochondria, and are mainly distributed in cardiac muscles, followed by liver tissues, skeletal muscles and kidneys. Significant elevation of transaminase is usually observed in acute damage of hepatocytes. In general, the sensitivity of ALT for identifying liver damage is higher than AST. However, levels of ALT and AST were not directly associated with the severity of liver damage (11). The results of the study were possibly associated with the relatively long course of the disease in the infants with cholestasis, which had already passed the acute phase and in which liver function may have been completely damaged. In addition, these results may be due to differences in individuals, different living environments and experimental error. To further confirm the results, an increased sample size and classification of the course of the disease are necessary.

Serum $\gamma$-GT is produced in the mitochondria of hepatocytes. Extrahepatic or intrahepatic obstruction during cholestasis blocks the excretion of $\gamma$-GT, thereby increasing the serum concentration of $\gamma$-GT (12). A number of studies have confirmed that serum $\gamma$-GT is an important biomarker to identify damage of hepatocytes and the degree of extrahepatic obstruction of bile ducts $(13,14)$. In the present study, serum $\gamma$-GT was found to be positively and significantly correlated with serum HA and serum cIV, suggesting that these three biomarkers were sensitive to cholestatic liver fibrosis. In addition, the results suggested that synthesis of $\gamma$-GT may be closely associated with the synthesis of HA and cIV. CHE is synthesized by liver tissue and secreted into blood. Its major physiological function is to catalyze the hydrolysis of acetylcholine. When the liver is damaged, synthesis of CHE and $\mathrm{CHE}$ activities are reduced. Reduction of serum CHE levels is closely associated with the pathological staging of liver fibrosis. Therefore, serum CHE can be used as a sensitive marker to determine the inflammatory changes and degree of damage in the liver parenchyma cells in the patients with chronic hepatitis. The higher the severity of liver fibrosis, the lower the serum levels of CHE (15). In the present study, cholestasis in the infants of the experimental group is a chronic process that gradually progresses to an advanced stage. Furthermore, serum CHE has been positively associated with serum cIV and $\mathrm{HA}$, to a certain extent reflecting close associations between the synthesis of CHE in the liver, as well as serum biomarkers (such as cIV and HA) of cholestatic liver fibrosis in infants. Further studies in the signal transduction pathways for the biosynthesis of CHE, cIV and HA will help determine if they share common characteristics. TBA is a bile acid that is not taken up by hepatocytes during intestinal reabsorption and is partially released into the blood circulation. Bile obstruction due to cholestasis, or secretion and/or ingestion impairment of hepatocytes, increases the serum TBA. A previous study has confirmed that serum bile acid is specific, sensitive and stable during liver functional changes (16). In the present study, serum TBA was negatively associated with serum HA and was statistically significant. Despite experimental errors that could not be eliminated, theoretically, the excessive production and accumulation of TBA may induce inflammation, thereby activating a series of intracellular signal transduction pathways. To date, the impact of TBA accumulation on HA synthesis has been rarely reported. Further studies are necessary in this regard. Although some of the results in the present study require further experimental confirmation, the study suggested that the serum levels of HA, cIV, $\gamma$-GT and CHE are sensitive markers for cholestatic liver fibrosis in infants.

In conclusion, statistically significant differences were observed for liver function markers (ALT, AST, TBIL, DBIL, IBIL, $\gamma$-GT and TBA) and biomarkers HA, PCIII and cIV of liver fibrosis between infants with cholestasis and healthy infants. Serum levels of HA, cIV, $\gamma$-GT and CHE are sensitive markers for cholestatic liver fibrosis in infants.

\section{Acknowledgements}

The study was supported by the Key Medical Science Research Program of Hebei Province (grant no. ZL20140195).

\section{References}

1. Elferink RO and Groen AK: Genetic defects in hepatobiliary transport. Biochim Biophys Acta 1586: 129-145, 2002

2. Benchimol EI, Walsh CM and Ling SC: Early diagnosis of neonatal cholestatic jaundice: Test at 2 weeks. Can Fam Physician 55: 1184-1192, 2009.

3. Dong $\mathrm{C}$ and Huang ZH: Diagnosis and treatment of cholestatic liver disease in infants. Zhonghua Linchuang Yishi Zazhi 40: $16-18,2012$.

4. Morgan NV, Hartley JL, Setchell KD, Simpson MA, Brown R, Tee L, Kirkham S, Pasha S, Trembath RC, Maher ER, et al: A combination of mutations in AKR1D1 and SKIV2L in a family with severe infantile liver disease. Orphanet J Rare Dis 8: 74, 2013.

5. Moyer V, Freese DK, Whitington PF, Olson AD, Brewer F, Colletti RB and Heyman MB; North American Society for Pediatric Gastroenterology, Hepatology and Nutrition: Guideline for the evaluation of cholestatic jaundice in infants: Recommendations of the North American Society for Pediatric Gastroenterology, Hepatology and Nutrition. J Pediatr Gastroenterol Nutr 39: 115-128, 2004.

6. Lee HH, Seo YS, Um SH, Won NH, Yoo H, Jung ES, Kwon YD, Park S, Keum B, Kim YS, et al: Usefulness of non-invasive markers for predicting significant fibrosis in patients with chronic liver disease. J Korean Med Sci 25: 67-74, 2010.

7. El-Shabrawi MH, Zein El Abedin MY, Omar N, Kamal NM, Elmakarem SA, Khattab S, El-Sayed HM, El-Hennawy A and Ali AS: Predictive accuracy of serum hyaluronic acid as a non-invasive marker of fibrosis in a cohort of multi-transfused Egyptian children with $\beta$-thalassaemia major. Arab J Gastroenterol 13: 45-48, 2012.

8. Nath NC, Rahman MA, Khan MR, Hasan MS, Bhuiyan TM, Hoque MN, Kabir MM, Raha AK and Jahan B: Serum hyaluronic acid as a predictor of fibrosis in chronic hepatitis $B$ and $C$ virus infection. Mymensingh Med J 20: 614-619, 2011.

9. Nobili V, Alisi A, Torre G, De Vito R, Pietrobattista A, Morino G De Ville De Goyet J, Bedogni G and Pinzani M: Hyaluronic acid predicts hepatic fibrosis in children with nonalcoholic fatty liver disease. Transl Res 156: 229-234, 2010.

10. Timpl R, Rohde H, Robey PG, Rennard SI, Foidart JM and Martin GR: Laminin - a glycoprotein from basement membranes. J Biol Chem 254: 9933-9937, 1979. 
11. Green RM and Flamm S: AGA technical review on the evaluation of liver chemistry tests. Gastroenterology 123: 1367-1384, 2002.

12. Liu D and Huang ZH: Differential diagnostic value of 5'-NT and $\gamma$-GT in infantile hepatitis syndrome (HIS) and biliary astresia. J Clin Exp Med 8: 16-17, 2009.

13. Tarantino G, Finelli C, Colao A, Capone D, Tarantino M, Grimaldi E, Chianese D, Gioia S, Pasanisi F, Contaldo F, et al: Are hepatic steatosis and carotid intima media thickness associated in obese patients with normal or slightly elevated gamma-glutamyl-transferase? J Transl Med 10: 50, 2012.
14. Tavian D, Degiorgio D, Roncaglia N, Vergani P, Cameroni I, Colombo R and Coviello DA: A new splicing site mutation of the $\mathrm{ABCB} 4$ gene in intrahepatic cholestasis of pregnancy with raised serum gamma-GT. Dig Liver Dis 41: 671-675, 2009.

15. Wang HL: Diagnostic value of serum ChE and TGF- $\beta$ combined detection in liver fibrosis. Pract Prev Med 16: 1237-11238, 2009.

16. Matsui S, Yamane T, Takita T, Oishi Y and Kobayashi-Hattori K: The hypocholesterolemic activity of Momordica charantia fruit is mediated by the altered cholesterol- and bile acid-regulating gene expression in rat liver. Nutr Res 33: 580-585, 2013. 